EPJ Web of Conferences 75, 02002 (2014)

DOI: $10.1051 /$ epjconf/ 20147502002

(c) Owned by the authors, published by EDP Sciences, 2014

\title{
Gilbert damping constant of FePd alloy thin films estimated by broadband ferromagnetic resonance
}

\author{
T. Kawai ${ }^{1}$, A. Itabashi ${ }^{1}$, M. Ohtake ${ }^{1}$, S. Takeda ${ }^{2}$, and M. Futamoto ${ }^{1}$ \\ ${ }^{1}$ Faculty of Science and Engineering, Chuo University, 1-13-27 Kasuga, Bunkyo-ku, Tokyo 112-8551, Japan \\ ${ }^{2}$ Magnontech Ltd., 787-16 Jurokken, Kumagaya, Saitama 360-0846, Japan
}

\begin{abstract}
Magnetic relaxation of FePd alloy epitaxial thin films with very flat surfaces prepared on $\mathrm{MgO}(001)$ substrate are measured by in-plane broadband ferromagnetic resonance (FMR). Magnetic relaxation is investigated as $\Delta \omega$ for FMR absorption peak by frequency sweep measurements. $\Delta H$ is calculated by using the measured $\Delta \omega$. Gilbert damping constant, $\alpha$, is estimated by employing a straight line fitting of the resonant frequency dependence of $\Delta H$. The $\alpha$ value for an FePd film deposited at $200{ }^{\circ} \mathrm{C}$, which shows disordered $A 1$ structure, is 0.010 and $\Delta H_{0}$, which is frequency independent part of $\Delta H$, is 10 Oe. The $\alpha$ value for a film annealed at $400{ }^{\circ} \mathrm{C}$, which shows partially $L 1_{0}$ ordered structure $(S=0.32)$, is 0.013 , which is slightly larger than that for the disorder $A 1$ structure film. However, $\Delta H_{0}$ for the annealed film is $85 \mathrm{Oe}$, which is much larger than that for the film with disordered structure. The results show that the magnetic relaxation of the $400{ }^{\circ} \mathrm{C}$ annealed film is mainly dominated by $\Delta H_{0}$, which is related with magnetic in-homogeneity caused by the appearance of perpendicular anisotropy of partially ordered phase.
\end{abstract}

\section{Introduction}

Spin-transfer switching is an attractive technique to deduce the electronic current for switching the magnetization direction of a magnetic element in devices like perpendicularly magnetized magnetoresistance random access memory (PMRAM) [1, 2]. For PMRAM devices, perpendicularly magnetized magnetic thin films with small Gilbert damping constant, $\alpha$, are required [1]. An $L 1_{0}$ ordered FePd alloy epitaxial thin film is one of candidate materials. Ferromagnetic resonance (FMR) is a powerful method in studying Gilbert damping constant, $\alpha$, of magnetic thin film. There are a few FMR studies of epitaxial FePd thin films with perpendicular magnetic anisotropy [3, 4]. It is reported that the line width of the uniform mode is found to be independent of frequency and hence departs from the linear frequency variation predicted by the Landau-Lifshitz-Gilbert (LLG) relaxation type [3]. However, it is well known that the magnetic relaxation in magnetic metal obeys mostly on the LLG type [5]. Therefore, it is interesting to investigate how the magnetic relaxation varies depending on the crystallographic ordering from disordered $A 1$ to the ordered $L 1_{0}$ state in FePd thin film.

In this study, Gilbert damping constant is estimated for $\mathrm{Fe}_{50} \mathrm{Pd}_{50}$ (at. \%) alloy epitaxial thin films with very flat surfaces, which are obtained on $\mathrm{MgO}(001)$ singlecrystal substrates by employing a two-step process; low- temperature deposition at $200{ }^{\circ} \mathrm{C}$ followed by hightemperature annealing at $400{ }^{\circ} \mathrm{C}$ [6]. Very flat surface is required in FMR measurements to evaluate intrinsic magnetic relaxation in order to avoid extrinsic effect by surface roughness [7]. Broadband FMR measurements using a VNA are carried out for 40nm thick films under static magnetic fields up to $1 \mathrm{kOe}$ in the film plane to measure magnetic relaxation. A difference in the magnetic relaxation between $\mathrm{FePd}$ films with a disordered $A 1$ structure and with a partially ordered $L 1_{0}$ structure is discussed.

\section{Experimental procedures}

FePd alloy thin films were prepared on polished $\mathrm{MgO}(001)$ single-crystal substrates by using a radiofrequency (RF) magnetron sputtering system equipped with a reflection high-energy electron diffraction (RHEED) facility. The base pressures were lower than 4 $\times 10^{-7} \mathrm{~Pa}$. The detail of sample preparation is reported in our previous paper [6]. FePd films of $40 \mathrm{~nm}$ thickness were deposited on $\mathrm{MgO}(001)$ substrates at $200^{\circ} \mathrm{C}$. Then, the films were annealed at 300 and $400{ }^{\circ} \mathrm{C}$ for $1 \mathrm{~h}$. The structural properties were investigated by RHEED and Xray diffraction (XRD) with $\mathrm{Cu}-\mathrm{K} \alpha$ radiation $(\lambda=$ $0.15418 \mathrm{~nm})$. Epitaxial growth of FePd film with $A 1(001)$ disordered structure was confirmed by RHEED for films deposited at $200{ }^{\circ} \mathrm{C}$. The epitaxial orientation

\footnotetext{
${ }^{\mathrm{a}}$ Corresponding author: kawai@futamoto.elect.chuo-u.ac.jp
} 
relationships were determined to be $\mathrm{FePd}(001)[100]_{A 1} \|$ $\mathrm{MgO}(001)[100]$ and $L 1_{0}[100] \| \mathrm{MgO}[100]$ by RHEED and XRD. The surface morphology was observed by AFM. The magnetization curves were measured by using a vibrating sample magnetometer. A VNA was used to measure dynamic magnetic property covering up to 10 $\mathrm{GHz}$, where RF magnetic field was applied orthogonally to the static magnetic field using a shorted micro-strip line $[7,8]$. The resonant frequency was determined as a frequency when an experimental complex permeability, $\mu$, showed a maximum value. Gilbert damping constant, $\alpha$, was estimated by using measured half line width between 1/2-power points for frequency sweep, $\Delta \omega$, as explained in the next section.

\section{Estimation method of Gilbert damping constant, $\alpha$, and perpendicular magnetic anisotropy}

The magnetic relaxation of magnetic thin films are usually discussed by using $\Delta \mathrm{H}$, which is full line width between 1/2-power points for magnetic field sweep FMR measurements, and is expressed in the following formula [9].

$\Delta H=\Delta H_{0}+\frac{4 \pi \alpha f_{r}}{\gamma}$,

where $\Delta H_{0}$, which is magnetic relaxation caused by inhomogeneity, is frequency independent part of $\Delta H, \alpha$ is Gilbert damping constant, $f_{\mathrm{r}}$ is resonant frequency, and $\gamma$ is gyromagnetic constant.

However, our FMR measurements are carried out not by magnetic field sweep but by frequency sweep. Then the measured value is not $\Delta H$ but $\Delta \omega$. Therefore, we need to calculate $\Delta H$ by using the measured $\Delta \omega$ employing the following conversion formula [9].

$$
\Delta H=\frac{2 \Delta \omega}{\gamma} \cdot \frac{1}{\sqrt{1+\frac{1}{4} \cdot\left(\frac{\omega_{m}}{\omega_{r}}\right)^{-}}}
$$

where $\quad \omega_{\mathrm{r}}=2 \pi f_{\mathrm{r}}, \quad \omega_{\mathrm{m}}=\gamma M_{\mathrm{s}} / \mu_{0}, \quad M_{\mathrm{s}} \quad$ is saturation magnetization, and $\mu_{0}$ is permeability of vacuum. $\alpha$ is calculated from the straight line fitting of the formula (1) with the measured $f_{\mathrm{r}}$ dependence of $\Delta H . \Delta H_{0}$ is defined as the intercept from the straight fitting. On the other hand, damping constant, $\alpha$, is also evaluated by fitting the calculated $\mu$ to each absorption peak of experimental $\mu \quad$ with best fitting parameters [7]. The line width of each absorption peak includes magnetic relaxation resulting from various factors. Therefore, the damping constant evaluated by this method is denoted as $\alpha_{\text {app }}$.

Perpendicular magnetic anisotropy field is determined using the following the Kittel resonant formula.

$$
\left(\frac{2 \pi f_{r}}{\gamma}\right)^{2}=H_{e x}-H_{\text {in }}-\left(H_{e x}+\frac{M_{S}}{\mu}-H_{\text {out }}\right) \text {, }
$$

where $H_{\mathrm{ex}}$ is applied static magnetic field, $H_{\mathrm{in}}$ is in-plane magnetic anisotropy field, and $H_{\text {out }}$ is perpendicular magnetic anisotropy field. $H_{\text {out }}$ is determined by fitting the formula (3) with the measured $H_{\mathrm{ex}}$ dependence of $f_{\mathrm{r}}$.

\section{Results and discussion}

Very flat surfaces are realized for the FePd films prepared by employing the two-step process. The properties of the films employed in the present study are listed in Table 1 [6]. Thin film samples with very flat surface with $R_{\mathrm{a}}$ less than $0.3 \mathrm{~nm}$ are obtained. $L 1_{0}$ order parameter, $S$, changes from 0 for an as-deposited film to 0.32 for a film annealed at $400{ }^{\circ} \mathrm{C}$. The magnetic easy axis is in-plane for all the films.

Table 1. Surface roughness, $R_{\mathrm{a}}, L 1_{0}$ order parameter, $S$, and magnetic anisotropy for FePd thin films prepared on $\mathrm{MgO}(001)$ substrate.

\begin{tabular}{|c|c|c|c|}
\hline Sample & As-deposited & $300^{\circ} \mathrm{C}$ annealed & $400^{\circ} \mathrm{C}$ annealed \\
\hline$R_{\mathrm{a}}(\mathrm{nm})$ & 0.22 & 0.15 & 0.18 \\
\hline Structure & $\begin{array}{c}A 1 \\
\text { (disorder) }\end{array}$ & $\begin{array}{c}A 1+L 1_{0} \\
\text { (partial order) }\end{array}$ & $\begin{array}{c}A 1+L 1_{0} \\
\text { (partial order) }\end{array}$ \\
\hline$S$ & 0 & 0.21 & 0.32 \\
\hline $\begin{array}{c}\text { Magnetic } \\
\text { anisotropy }\end{array}$ & In-plane & In-plane & In-plane \\
\hline
\end{tabular}

In-plane magnetization curves are shown in Fig. 1. The easy magnetization axis is along $<100>$ for all three films. The anisotropy fields of as-deposited and $300{ }^{\circ} \mathrm{C}$ annealed films are almost similar at $30 \mathrm{Oe}$, whereas that of $400{ }^{\circ} \mathrm{C}$ annealed film is 70 Oe which is larger than the other two films. In-plane magnetic anisotropy seems to have varied with increasing partial ordering of $L 1_{0}$ structure. The coercivity is also changed from $10 \mathrm{Oe}$ for the as-deposited film to 24 Oe for the $400{ }^{\circ} \mathrm{C}$ annealed film which suggests that domain wall motions are suppressed in the $400{ }^{\circ} \mathrm{C}$ annealed film. As shown in Table 1, small partial order $(\mathrm{S}=0.21)$ is developed even in the $300{ }^{\circ} \mathrm{C}$ annealed film, but such small partial order shows no effect on static in-plane magnetic property.

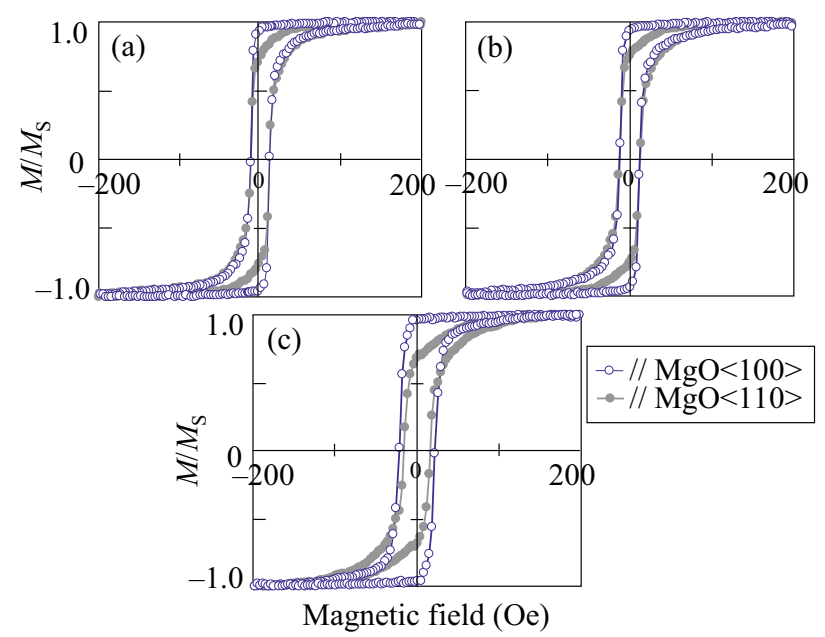

Fig. 1 In-plane magnetization curves measured for (a) FePd film deposited on $\mathrm{MgO}(001)$ at $200{ }^{\circ} \mathrm{C}$, and $\mathrm{FePd}$ films annealed at (b) $300{ }^{\circ} \mathrm{C}$ and (c) $400{ }^{\circ} \mathrm{C}$ after deposition.

The examples of measured complex permeability for three FePd films are shown in Fig. 2, where applied static magnetic filed is 174 Oe along $<100>$. Clear Lorenz type absorption peaks are observed for all samples. The 
permeability values for the $400{ }^{\circ} \mathrm{C}$ annealed film are small compared with those of the other two films. This is possibly due to the enhanced in-plane anisotropy field (70 Oe) of the $400{ }^{\circ} \mathrm{C}$ annealed film compared with the other films (30 Oe).

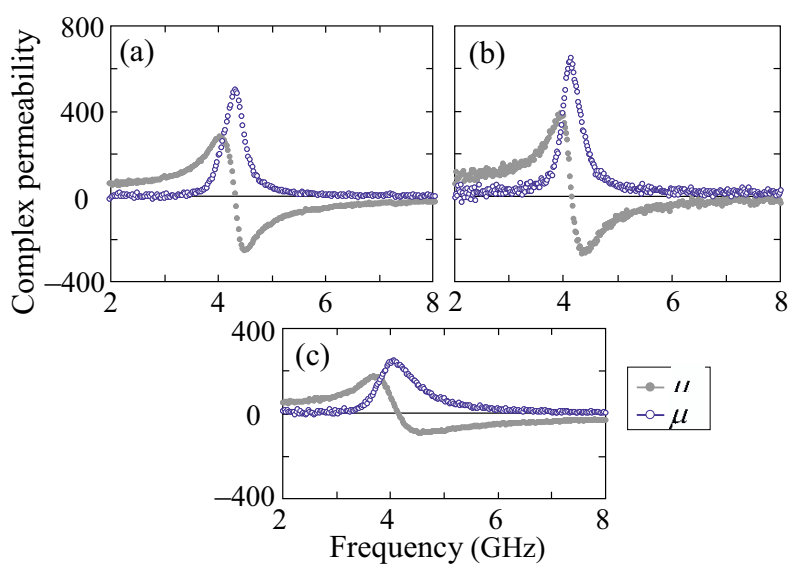

Fig.2 Examples of complex permeability measured for (a) FePd film deposited on $\mathrm{MgO}(001)$ at $200{ }^{\circ} \mathrm{C}$, and $\mathrm{FePd}$ films annealed at (b) $300{ }^{\circ} \mathrm{C}$ and (c) $400{ }^{\circ} \mathrm{C}$ after deposition.

Complex permeability measurements are carried out by changing the external static magnetic field from 0 to 1 kOe. Examples of absorption peaks are shown in Fig. 3. Resonant frequency is defined at the frequency where $\mu$ shows a maximum value. Solid lines show the calculated $\mu$ using best fitting parameters of $M_{\mathrm{s}}, K_{1}$, and $\alpha$ [7], where $g$-factor is fixed at 2.07 [2]. There are very good agreements between the experiments and the calculations for the as-deposited film and the film annealed at $300{ }^{\circ} \mathrm{C}$ for the applied static magnetic field range, whereas for the film annealed at $400{ }^{\circ} \mathrm{C}$, good agreements are recognized only for more than $300 \mathrm{Oe}$. As shown in Fig. 1, magnetic saturation is already reached at $200 \mathrm{Oe}$ in the static magnetic field characteristic, but the FMR measurement indicates that more than 300 Oe magnetic field is required to excite the spin wave in the uniform mode in radio frequency region.

Figure 4 shows the static magnetic field dependence of resonant frequency, $f_{\mathrm{r}}$, for three FePd thin films. Solid lines are the fitting curves by formula (3) using best fitting parameters of $M_{\mathrm{s}}, H_{\mathrm{in}}$, and $H_{\text {out }}$. There is no difference of magnetic field dependence of $f_{\mathrm{r}}$ between for the as-deposited film and for the $300{ }^{\circ} \mathrm{C}$ annealed film, which suggests that any observable perpendicular anisotropy is not developed in the $300{ }^{\circ} \mathrm{C}$ annealed film though a small partial $L 1_{0}$ structural ordering is observed. The perpendicular anisotropy field is estimated to be 1.5 kOe by the fitting for the $400{ }^{\circ} \mathrm{C}$ annealed film.

The $\alpha_{\text {app }}$ values obtained by the fitting for each absorption peak are shown in Fig. 5 as a function of resonant frequency reciprocal, $1 / f_{\mathrm{r}}$. The $\alpha_{\text {app }}$ values tend to become small as the frequency reciprocal, $1 / f_{\mathrm{r}}$, becomes small. The $\alpha_{\text {app }}$ values are almost similar for the as-deposited and for the $300{ }^{\circ} \mathrm{C}$ annealed films, whereas $\alpha_{\text {app }}$ is large for the $400{ }^{\circ} \mathrm{C}$ annealed film compared with other films. Gilbert damping constant, $\alpha$, defined in the LLG equation should be frequency independent. Therefore, we tried to separate the measured magnetic

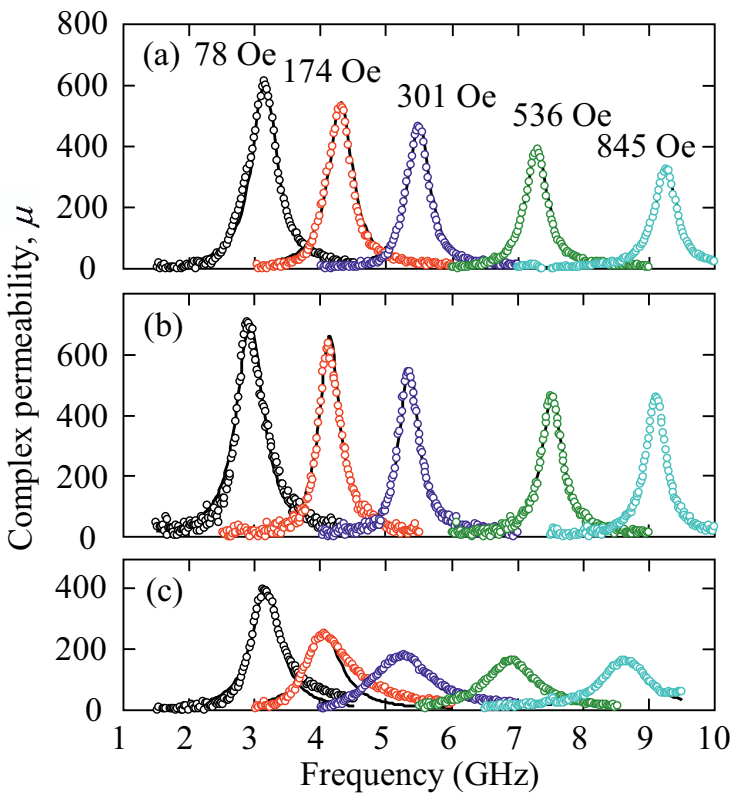

Fig. 3 Complex permeability measured under various static magnetic fields for (a) the $\mathrm{FePd}$ film deposited on $\mathrm{MgO}(001)$ at $200{ }^{\circ} \mathrm{C}$, and $\mathrm{FePd}$ films annealed at (b) $300{ }^{\circ} \mathrm{C}$ and (c) $400{ }^{\circ} \mathrm{C}$ after deposition. Solid lines are fitting curves.

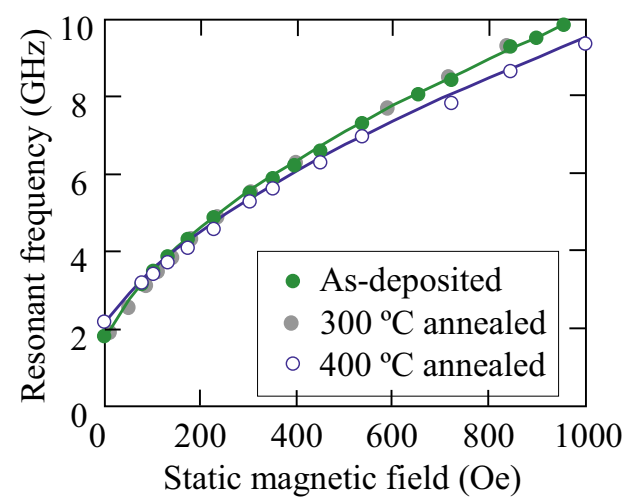

Fig. 4 Static magnetic field dependence of resonant frequency for (a) the $\mathrm{FePd}$ film deposited on $\mathrm{MgO}(001)$ at $200{ }^{\circ} \mathrm{C}$, and FePd films annealed at (b) $300{ }^{\circ} \mathrm{C}$ and (c) $400{ }^{\circ} \mathrm{C}$ after deposition. Circles are experiments and solid lines are calculations.

relaxation into the frequency independent part and the frequency dependent part as denoted in formula (1) using the measured $\Delta \omega . \Delta \omega$ is defined by a parabolic fitting using the reciprocal of $\mu$ near the resonance peak. $\Delta H$ is calculated by formula (2) using the measured $\Delta \omega$. Frequency dependences of $\Delta H$ are shown in Fig.6. Gilbert damping constant, $\alpha$, is calculated by using the slope of the straight line and $\Delta H_{0}$, which means $\Delta H$ caused by inhomogeneous effect, is defined as an intercept at y-axis. These Gilbert damping constants, $\alpha$, and $\Delta H_{0}$ values are shown in the insets of the figure. $\Delta H$ values for the as-deposited and for the $300{ }^{\circ} \mathrm{C}$ annealed films are almost similar. However, $\Delta H$ values for the 400 ${ }^{\circ} \mathrm{C}$ annealed film are much larger than those of other films.

The $\alpha$ value for the as-deposited film is 0.010 and $\Delta H_{0}$ is 10 Oe. The $\alpha$ value for the $400{ }^{\circ} \mathrm{C}$ annealed film is 0.013 , which is slightly larger than that for the asdeposited film. However, $\Delta H_{0}$ for the annealed film is 


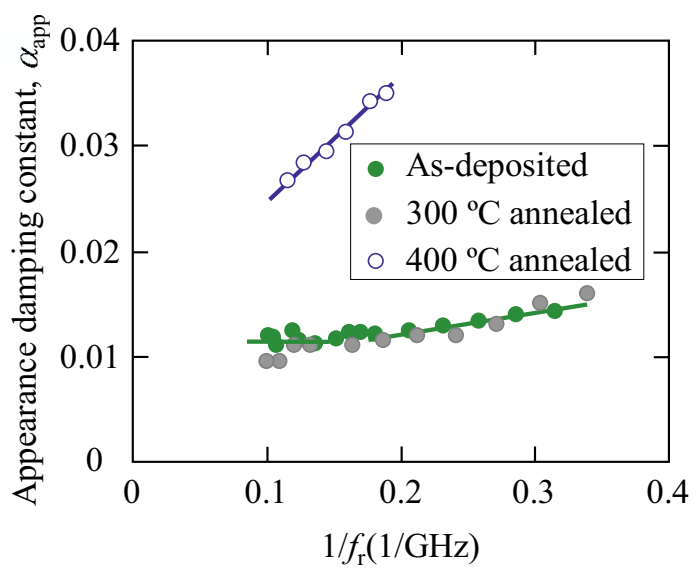

Fig. 5 Appearance damping constant, $\alpha_{\mathrm{app}}$, as a function of $1 / f_{\mathrm{r}}$ for (a) the FePd film deposited on $\mathrm{MgO}(001)$ at $200{ }^{\circ} \mathrm{C}$, and FePd films annealed at (b) $300{ }^{\circ} \mathrm{C}$ and (c) $400{ }^{\circ} \mathrm{C}$ after deposition.

$85 \mathrm{Oe}$, which is much larger than that for the as-deposited film. The results suggest that the magnetic relaxation of partially $L 1_{0}$ ordered FePd film with perpendicular anisotropy is mainly dominated by $\Delta H_{0}$ which is caused by magnetic in-homogeneity in the film. As shown in figures 1,4 and 5 , there is little difference in the magnetic relaxation between the as-deposited film $(\mathrm{S}=0)$ and the $300{ }^{\circ} \mathrm{C}$ annealed film with partial $L 1_{0}$ ordering $(\mathrm{S}=0.21)$. The result indicates that the small structural inhomogeneity without perpendicular anisotropy shows almost no effect on magnetic relaxation but the magnetic in-homogeneity caused by the appearance of perpendicular anisotropy shows significant effect. Such magnetic in-homogeneity may cause frequency independent relaxation like two magnon process [10].

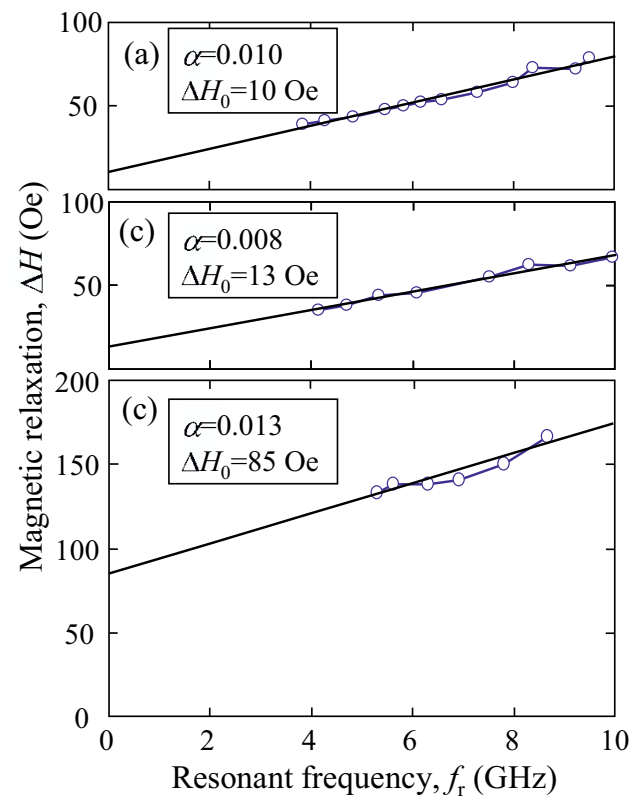

Fig. 6 Resonant frequency dependence of $\Delta H$ for (a) FePd film deposited on $\mathrm{MgO}(001)$ at $200{ }^{\circ} \mathrm{C}$, and $\mathrm{FePd}$ films annealed at (b) $300{ }^{\circ} \mathrm{C}$ and (c) $400{ }^{\circ} \mathrm{C}$ after deposition. Solid lines are straight line fittings.

\section{Conclusions}

Magnetic relaxation of FePd epitaxial thin films with very flat surfaces is measured by broadband FMR. Measured magnetic relaxation is evaluated by separating into a frequency independent part, $\Delta H_{0}$, and a frequency dependent part. Gilbert damping constant, $\alpha$, is calculated by using the slope of frequency dependent part. The $\alpha$ value for the $\mathrm{FePd}$ film deposited at $200{ }^{\circ} \mathrm{C}$, which shows disorder $A 1$ structure, is 0.010 and $\Delta H_{0}$, is 10 Oe. Meanwhile, the $\alpha$ value for the $400{ }^{\circ} \mathrm{C}$ annealed film, which shows partially $L 1_{0}$ ordered structure $(S=0.32)$, is 0.013 , which is slightly larger than that of the disorder $A 1$ structure film. However, $\Delta H_{0}$ for the $400{ }^{\circ} \mathrm{C}$ annealed film is $85 \mathrm{Oe}$, which is much larger than that for the disorder $A 1$ structure film. The results show that the magnetic relaxation of partially $L 1_{0}$ ordered FePd film is mainly dominated by $\Delta H_{0}$, which is related with magnetic in-homogeneity developed by perpendicular anisotropy in the film during ordering process.

\section{Acknowledgements}

We wish to thank Hitachi Metals Ltd. for supporting this work. We also thank Dr. H. Suzuki of KEYCOM Corp. for his kind advice in the broadband FMR measurements.

\section{References}

1. J. C. Slonczewski, J. Magn. Magn. Matter. 159, L1 (1996).

2. L. Berger, Phys. Rev. B 54, 9353 (1996).

3. J. BenYoussef, H. Le Gall, N. Vukadinovic, V. Gehanno, A. Marty, Y. Samson, and B. Gilles, J. Magn. Magn. Matter. 202, 277 (1999).

4. N. Vukadinovic, H. Le Gall, J. Ben Youssef, V. Gehanno, A. Marty, Y. Samson, and B. Gilles, Eur. Phys. J. B 13, 445 (2000).

5. B. Heinrich, J.F. Cochran, and R. Hasegawa, J. Appl. Phys. 57, 3690 (1985).

6. A. Itabashi, M. Ohtake, S. Ouchi, F. Kirino, and M Futamoto, IEEE, Trans. Magn., 48, 3203 (2012).

7. T. Kawai, Y. Asai, M. Ohtake, S. Takeda, and M. Futamoto, EPJ Web of Conferences 40, 13001 (2013).

8. S. Takeda and H. Suzuki, J. Magn. Soc. Jpn., 33, 171 (2009).

9. S. S. Kalarickal, P. Krivosik, M. Wu, and C. E. Patton, J. Appl. Phys. 99, 093909 (2006).

10. M. Sparks, Ferromagnetic relaxation, McGraw Hill p.163, (1964) 